\title{
Tumour-suppressor microRNAs let-7 and mir-101 target the proto-oncogene MYCN and inhibit cell proliferation in MYCN-amplified neuroblastoma
}

\author{
J Buechner ${ }^{1,2}$, E Tømte ${ }^{2}$, BH Haug ${ }^{2}$, JR Henriksen², C Løkke², T Flægstad ${ }^{1,2}$ and C Einvik ${ }^{*, 1,2}$ \\ 'Department of Paediatrics, University Hospital of North-Norway, 9038 Tromsø, Nonway; ${ }^{2}$ Paediatric Research Group, Department of Clinical Medicine, \\ Faculty of Health Sciences, University of Tromsø, 9037 Tromsø, Norway
}

BACKGROUND: MicroRNAs (miRNAs) regulate expression of many cancer-related genes through posttranscriptional repression of their mRNAs. In this study we investigate the proto-oncogene MYCN as a target for miRNA regulation.

METHODS: A luciferase reporter assay was used to investigate software-predicted miRNA target sites in the $3^{\prime}$-untranslated region ( $3^{\prime} U T R$ ) of MYCN. The miRNAs were overexpressed in cell lines by transfection of miRNA mimics or miRNA-expressing plasmids. Mutation of the target sites was used to validate MYCN 3'UTR as a direct target of several miRNAs. To measure miRNA-mediated suppression of endogenous $\mathrm{N}$-myc protein, inhibition of proliferation and inhibition of clonogenic growth, miRNAs were overexpressed in a MYCN-amplified neuroblastoma cell line.

RESULTS: The results from this study show that MYCN is targeted by several miRNAs. In addition to the previously shown mir-34a/c, we experimentally validate mir-449, mir-19a/b, mir-29a/b/c, mir-101 and let-7e/mir-202 as direct MYCN-targeting miRNAs. These miRNAs were able to suppress endogenous N-myc protein in a MYCN-amplified neuroblastoma cell line. The let-7e and mir-202 were strong negative regulators of MYCN expression. The mir-101 and the let-7 family miRNAs let-7e and mir-202 inhibited proliferation and clonogenic growth when overexpressed in Kelly cells.

CONCLUSION: The tumour-suppressor miRNAs let-7 and mir-101 target MYCN and inhibit proliferation and clonogenic growth of MYCN-amplified neuroblastoma cells.

British Journal of Cancer (201 I) 1 05, 296-303. doi:I0.1038/bjc.201 I.220 www.bjcancer.com

Published online 7 June 2011

(C) 201। Cancer Research UK

Keywords: neuroblastoma; N-myc; MYCN; let-7; mir-101; 3'UTR

The transcription factor N-myc, which is encoded by the human $M Y C N$ proto-oncogene, belongs to the Myc family of DNA binding basic region/helix-loop-helix/leucine zipper (bHLHZip) proteins in which c-Myc, L-Myc and N-myc are the best characterised members (Meyer and Penn, 2008). The genomic sequences of $M Y C N$ and $c-M Y C$ share wide structural homology. Both genes consist of three exons, where the first exon is untranslated and exons 2 and 3 encode the translated regions (Kohl et al, 1986). $\mathrm{N}$-myc and c-Myc proteins are of similar sizes (464 aa and 454 aa, respectively). However, the MYCN mRNA is longer, mainly because of a larger $3^{\prime}$-untranslated region ( $3^{\prime}$ UTR). In addition to structural and sequence homologies within the Myc family, the functions of these proteins are closely related. Myc proteins heterodimerise with the bHLHZip-protein Max to a transcription factor complex that binds to specific E-box DNA motifs $\left(5^{\prime}\right.$-CACGTG- $3^{\prime}$ or variants thereof) and activates transcription of genes involved in diverse cellular functions, including cell growth and proliferation, metabolism, apoptosis and differentiation (Bell et al, 2010; Larsson and Henriksson, 2010). In addition to Myc,

*Correspondence: Dr C Einvik; E-mail: christer.einvik@uit.no Received 28 March 201 I; revised 13 May 20I I; accepted 17 May 20I I; published online 7 June 2011
Max also dimerises with the bHLHZip-proteins Mad/Mnt. These complexes also bind to E-box elements, but repress transcription through the recruitment of corepressors (Nilsson and Cleveland, 2003). The N-myc protein has recently been shown to repress TrkA and $p 75 N T R$ expression by interaction with $\mathrm{Sp} 1$ and Miz-1 at proximal/core promoter regions. In this repression complex, $\mathrm{N}$-myc recruited the histone deacetylase HDAC1 to silence gene expression by deacetylating chromatin at the promoter (Iraci et al, 2011). Similar transcriptional repression by $\mathrm{N}$-myc has also been shown at an Sp1-binding site in the tissue transglutaminase (TG2) core promoter (Liu et al, 2007).

Given the fundamental role of Myc proteins on cellular processes, their activity in nontransformed cells needs to be spatially and timely controlled. Although $c-M Y C$ is expressed during all developmental stages and in a distinct pattern throughout the cell cycle of dividing cells (Hooker and Hurlin, 2006), MYCN expression is restricted mainly to the peripheral and central nervous system and epithelial cells during particular embryonal stages (Stanton et al, 1992). Expression is controlled at multiple levels, including gene transcription through upstream regulators, mRNA turnover, and protein activation or decay upon phosphorylation of specific protein residues (Meyer and Penn, 2008).

Dysregulation of Myc activity is an oncogenic hallmark in human malignancies. Activation of Myc is mainly caused by gene 
translocations or amplifications, or enhanced protein translation or stability, leading to overexpression of a structural normal protein (Vita and Henriksson, 2006; Albihn et al, 2010). Neuroblastoma is a common childhood solid tumour in which MYCN is amplified in $\sim 15 \%$ of cases. MYCN amplification (MNA) in the tumour is closely related to poor survival of the patients, despite all modern multi-modal treatment efforts (Maris et al, 2007; Maris, 2010). In contrast, non-amplified (non-MNA), lowstage neuroblastoma tumours have the propensity to differentiate into benign subtypes, or regress spontaneously. Paradoxically, these localised, low-risk tumours also have elevated N-myc activity. Their MYCN mRNA and protein levels exceed the levels of high-risk non-MNA tumours with poor outcome, but do not reach those of MNA tumours (Cohn et al, 2000; Tang et al, 2006; Westermann et al, 2008). It has therefore been supposed that neuroblastoma cells with moderately elevated N-myc sustain the capacity to undergo apoptosis and neuronal differentiation (Edsjo et al, 2004).

Despite its clinical significance, it is still largely unknown how $M Y C N$ expression is regulated in neuroblastoma. Here, we have investigated how microRNAs (miRNAs) contribute to the control of MYCN expression in MNA neuroblastoma cells. MiRNAs are a class of small (19-22 nt), non-coding RNA molecules that repress protein expression through imperfect binding to sequences in the $3^{\prime} \mathrm{UTR}$ of target mRNAs. Most miRNAs are transcribed as long monocistronic, bicistronic or polycistronic primary transcription units (pri-miRNAs) by RNA polymerase II, and cleaved by a series of cellular processing events to produce mature miRNAs (Siomi and Siomi, 2009). The degree of complementarity between mature miRNAs and target mRNAs determines the mechanism responsible for blocking protein synthesis. In mammals, miRNA - mRNA interactions are most often through imperfect base pairing, resulting in translational repression. It has been estimated that $30 \%$ of all human genes are regulated by miRNAs (Lewis et al, 2005).

In the recent years, several studies have been carried out to investigate how $\mathrm{N}$-myc as a transcription factor affects expression of miRNAs (Stallings et al, 2010). On the contrary, only little is known about $M Y C N$ as a miRNA target. The tumour-suppressor miRNA mir-34a has been experimentally validated to directly target the $3^{\prime} \mathrm{UTR}$ sequence of MYCN (Wei et al, 2008). In addition, a miRNA binding site for mir-101 has also been reported in the $M Y C N-3^{\prime}$ UTR sequence (Lewis et al, 2005). However, a more systematic screening for miRNA binding sites and validation studies of miRNA/MYCN-3'UTR interactions have to our knowledge not been performed. It is well established that cellular proto-oncogenes are regulated by miRNAs, and that disturbances in these relations contribute to cancer development.

In this study, we have investigated the $3^{\prime} \mathrm{UTR}$ of the MYCN proto-oncogene for conserved miRNA binding sites. We established several miRNAs as MYCN-controlling miRNAs, and define a subset with antiproliferative and anticlonogenic properties.

\section{MATERIALS AND METHODS}

\section{Cell lines}

The MNA neuroblastoma cell lines SMS-KCN, SMS-KCNR, SMSKANR, SK-N-BE(2), Kelly and the non-MNA cell line SK-N-AS were grown in RPMI-1640 medium, MNA IMR-32 and LAN5 in DMEM medium with $1 \%$ NEAA and $2 \mathrm{~mm}$ glutamine, and nonMNA SH-SY-5Y in HAM-F12 with $1 \%$ NEAA and $2 \mathrm{~mm}$ glutamine. The embryonic kidney cell line HEK293 was grown in DMEM. All media were supplemented with 10\% FBS. Cells were maintained in a humidified $37^{\circ} \mathrm{C}$ incubator with $5 \% \mathrm{CO}_{2}$, supplied with fresh complete medium every 3 days, and sub-cultured before confluence was reached.

\section{Genomic DNA from patient samples and neuroblastoma} cell lines

Genomic DNA samples from 39 primary neuroblastoma tumours (34 MNA and 5 non-MNA) were kindly provided by Professor T Martinsson (Gothenborg University, Sweden) after informed consent from the patients and ethical approval by the institution. Genomic DNA from neuroblastoma cell lines was isolated with the DNeasy Blood and Tissue kit (Qiagen, Crawley, UK) according to the manufacturer's protocol.

\section{Screening for mutations in the $M Y C N 3^{\prime} \mathrm{UTR}$}

The MYCN $3^{\prime}$ UTR in each DNA sample was amplified in a $50 \mu \mathrm{l}$ PCR reaction, using $25 \mathrm{ng}$ genomic DNA, Platinum Taq polymerase (Invitrogen, Carlsbad, CA, USA) and $10 \mathrm{~nm}$ primers (Supplementary Table 1). Bi-directional sequencing was performed in $10 \mu \mathrm{l}$ reactions, using $50 \mathrm{ng}$ of purified $M Y C N 3^{\prime} \mathrm{UTR}$, BigDye3.1 reagent (Applied Biosystems, Carlsbad, CA, USA) and $10 \mathrm{~nm}$ forward or reverse primer. Sequences were analysed on an ABI Prism (Applied Biosystems) using the in-house Sequencing Core Facility.

\section{Exogenously overexpression of miRNAs}

The sequences for pre-mir-34a, $-34 c$ and $-106 b$ flanked by $250 \mathrm{nt}$ genomic sequence in both directions were amplified from SK-N$\mathrm{BE}(2)$ genomic DNA using Platinum Taq polymerase (Invitrogen) and primers as described in Supplementary Table 1. Pre-mir-449a and $-449 b$, which are expressed from the mir-449 cluster, were amplified from SK-N-BE(2) DNA as a bi-cistronic mir-449a/449b sequence ('mir-449ab'). The PCR products were cloned into the MCS of the expression vector pcDNA6.2-EmGFP (Invitrogen) by In-Fusion cloning (Clontech Laboratories, Mountain View, CA, USA) according to the manufacturer's recommendations. Bi-directional DNA sequencing verified all vectors. All other miRNAs used in this study were purchased as miRNA mimics (Shanghai GenePharma, Shanghai, China) as listed in Supplementary Table 1. Overexpression of miRNAs was confirmed by miRNA-specific RT-qPCR as described (Buechner et al, 2011).

As negative controls, we used pcDNA6.2-EmGFP containing pre-mir-346 in vector-based miRNA experiments and the Negative Control mimic (Shanghai GenePharma) for mimic-based expression.

\section{Luciferase/MYCN-3'UTR expression constructs}

To generate the pMIR-MYCN-UTR vector, the full-length MYCN3'UTR sequence was amplified from genomic DNA and cloned into the MCS of the Firefly luciferase expressing pMIR-REPORT (Ambion, Austin, TX, USA). QuikChange Multi Site-directed Mutagenesis Kit (Stratagene, La Jolla, CA, USA) was used to specifically mutate individual miRNA seed sequences. To disrupt miRNA binding, a two-base mismatch within position $2-6$ of the corresponding $M Y C N-3^{\prime}$ UTR seed sequence was introduced (Doench and Sharp, 2004) (Supplementary Figure 1). To ensure complete disruption of miRNA seed sequences in selected nonrescued cases, we extended the mutations to include a complete seed mismatch (position 2-8). Mutagenesis was performed according to the Stratagene's standard protocol. All mutagenesis primers are listed in Supplementary Table 1. All mutations were confirmed by bi-directional sequencing.

\section{Luciferase reporter assay (LRA)}

LRAs were performed as previously described (Henriksen et al, 2011). Briefly, HEK293 cells were co-transfected with $20 \mathrm{ng}$ pGL4.75[hRluc/CMV] (Promega, Madison, WI, USA), $2900 \mathrm{ng}$ miRNA expression vector or $0.43 \mu \mathrm{l}$ miRNA mimic $(20 \mu \mathrm{M})$, and $100 \mathrm{ng}$ of either wild-type pMIR-MYCN-UTR or a mutant variant. 
At $48 \mathrm{~h}$ hours after transfection, Renilla and Firefly luciferase activities were analysed using the Dual Luciferase Assay (Promega). Each miRNA transfection was done in triplets and independently repeated at least three times, resulting in at least nine LRAs for each individual miRNA. Luciferase activities were analysed in duplicates. Normalisation included two steps: first, the Firefly luciferase activity was normalised to the Renilla luciferase activity, and second, the normalised luciferase activity of transfected NC (pre-mir-346 or Negative Control mimic) was set as relative luciferase activity of 1 . The PASW Statistics 18 software (SPSS Inc., Chicago, IL, USA) was used for data analyses and boxplot charts.

\section{Western blotting}

Cells were transfected in six-well plates with $5 \mu \mathrm{l}$ miRNA mimic $(20 \mu \mathrm{M})$ using a standard Lipofectamine2000 reverse transfection protocol (Invitrogen). After $48 \mathrm{~h}$, protein expression was determined by western blotting as previously described (Henriksen et al, 2011) with primary antibodies against $\mathrm{N}$-myc $(1: 500$, Calbiochem/Merck, Darmstadt, Germany), $\beta$-Actin (1:1250, Sigma-Aldrich Corp., St Louis, MO, USA), and the secondary antibodies IRDye800CW $(1: 5000$, Rockland, Gilbertsville, PA, USA) and Alexa Fluor 680 (1:5000, Invitrogen).

\section{Cell proliferation assay}

Cell growth was monitored continuously in 16-well E-plates on the xCELLigence system (Roche, Mannheim, Germany). Kelly cells were seeded in $160 \mu \mathrm{l}$ media (15000 cells per well) and transfected in triplicates $4-6 \mathrm{~h}$ later with $60 \mu \mathrm{l}$ of a transfection mix containing $0.2 \mu \mathrm{l}$ Lipofectamine 2000 and $0.6 \mu \mathrm{l}$ miRNA mimic $(20 \mu \mathrm{M})$. Proliferation was recorded automatically as cell index every $30 \mathrm{~min}$ for a minimum of $72 \mathrm{~h}$. The cell index is derived from changes in electrical impedance as the cells interact with interdigitated microelectrodes integrated on the bottom of the E-plate.

\section{Clonogenic cell assay}

To investigate the capability of single cells to survive and proliferate after transfection of specific miRNA mimics, cells were transfected as described and seeded in six-well-dishes in a density of 200 cells per well. Staining was performed as previously described (Henriksen et al, 2011).

\section{RESULTS}

\section{Mutational screening of the MYCN $3^{\prime} \mathrm{UTR}$}

In order to reveal mutations or single-nucleotide polymorphisms (SNPs) that could potentially disturb miRNA-mediated suppression of MYCN expression in neuroblastoma, the entire $M Y C N$ $3^{\prime}$ UTR sequence from 7 MNA neuroblastoma cell lines (SMS-KCN, SMS-KCNR, SMS-KANR, SK-N-BE(2), Kelly, IMR-32 and LAN5) and 39 neuroblastoma primary tumours ( $34 \mathrm{MNA}$ and 5 nonMNA) was sequenced. According to the human MYCN germ-line sequence (Genbank accession no. NM_005378) six cell lines contained the wild-type $M Y C N-3^{\prime} U T R$ sequences, whereas the LAN5 cell line was found to carry a homozygous point mutation at position 250, changing a cytosine to a thymine (C250T) (Figures $1 \mathrm{~A}$ and $\mathrm{B})$. The identical homozygous mutation was found in $35 \%$ of MNA primary tumours (12 of 34 ) and $20 \%$ of non-MNA primary tumours ( 1 of 5 ). The $\mathrm{C} / \mathrm{T}$ variation at this genomic position is termed rs922 in the NCBI RefSNP database. We were not able to detect heterozygous variants at this position, or any other mutations in the MYCN-3'UTR sequence.

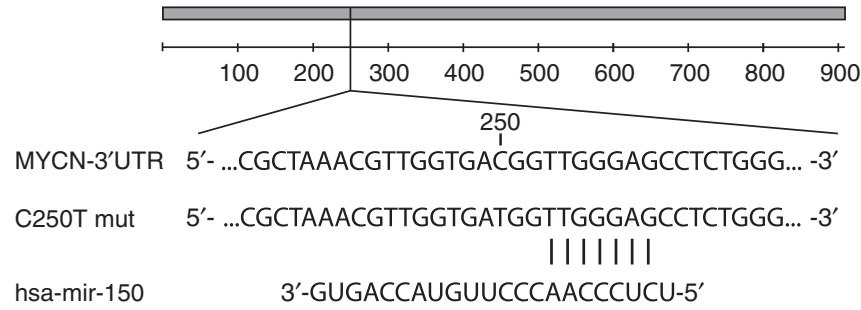

B

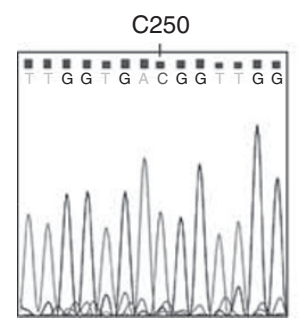

MYCN 3'UTR wt

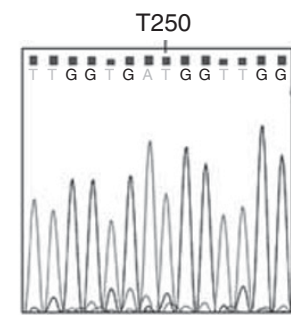

MYCN 3'UTR C250T
Figure I $C$ to $T$ variation at position 250 in the MYCN $3^{\prime} \cup T R$. Mutational analysis of the human MYCN 3'UTR (NM_005378). (A) Schematic overview of the MYCN 3'UTR including the rs922 SNP (C250T mut) located $3 \mathrm{bp}$ upstream of the predicted target sequence for mir-I50. (B) Exclusively homozygous $\mathrm{C} / \mathrm{C}$ or $\mathrm{T} / \mathrm{T}$ genotype variants were detected in neuroblastoma cell lines and primary tumours.

\section{Prediction of miRNAs targeting the MYCN $3^{\prime}$ UTR}

As a first step to identify $M Y C N$-targeting miRNAs, we combined three miRNA target prediction programmes, TargetScan 5.1 (Lewis et al, 2003), MiRanda (John et al, 2004) and PicTar (Lall et al, 2006), to predict potential miRNA binding sites in the full-length $M Y C N-3^{\prime}$ UTR sequence. TargetScan, which uses site and miRNA conservation across different species as selection criteria, predicted 43 individual miRNAs with broad conservation among vertebrates, targeting a total of 14 different conserved miRNA binding sites. We intersected the TargetScan miRNA seed subset with the predictions from the MiRanda and PicTar programmes. Of the 14 conserved binding sites predicted by TargetScan, 13 were also predicted by one of the other programmes (Figure 2A). To completely cover these 13 binding sites, we selected 20 individual miRNAs for experimental validation (Supplementary Table 2). We finally expanded the panel to include mir-202, a let-7 miRNA family member conserved only among mammals (Figure $2 \mathrm{~B}$ ).

\section{Experimental validation of $M Y C N-3^{\prime} \mathrm{UTR}$ target sites by LRAs}

We used LRA to experimentally validate whether the 21 candidate miRNAs were able to target the $M Y C N-3^{\prime} \mathrm{UTR}$ sequence. Briefly, the full-length MYCN 3'UTR (909 bp) was cloned into the pMIRReport vector downstream of the luciferase gene, generating pMIR-MYCN-UTR. This vector was co-transfected with either miRNA mimics or miRNA-expression plasmids into HEK293 cells, and luciferase activity was measured and compared with cotransfections with a negative control miRNA. Overexpression of miRNAs was confirmed by qRT - PCR assays (data not shown). As shown in Figure 2C, 14 individual miRNAs (representing 9 of the 13 predicted miRNA binding sites) reduced the normalised luciferase activity by $>20 \%$. These miRNAs belong to six distinct miRNA seed families: the mir-34abc/449abc/699, mir-19a/b, mir-29abc, mir-101, let-7/98/202, and mir-17/20/93/106/519 family.

To investigate if the observed reduction in luciferase activity was caused by direct miRNA/MYCN-3'UTR interactions, we introduced point mutations into the seed sequences of the nine potential 
A

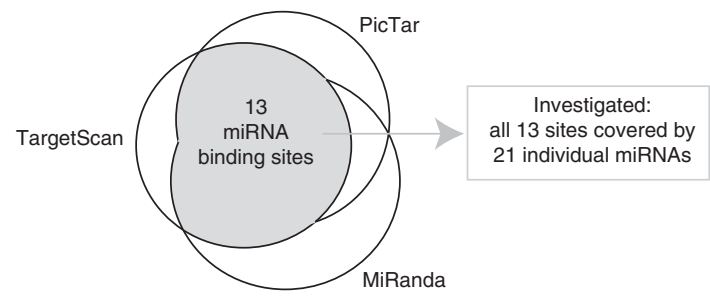

B

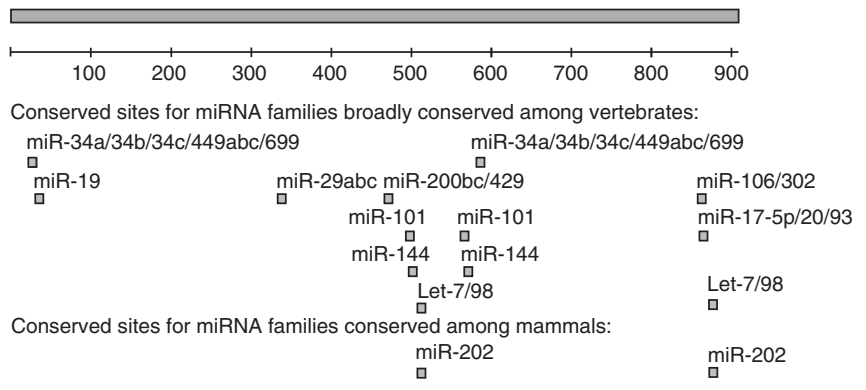

C

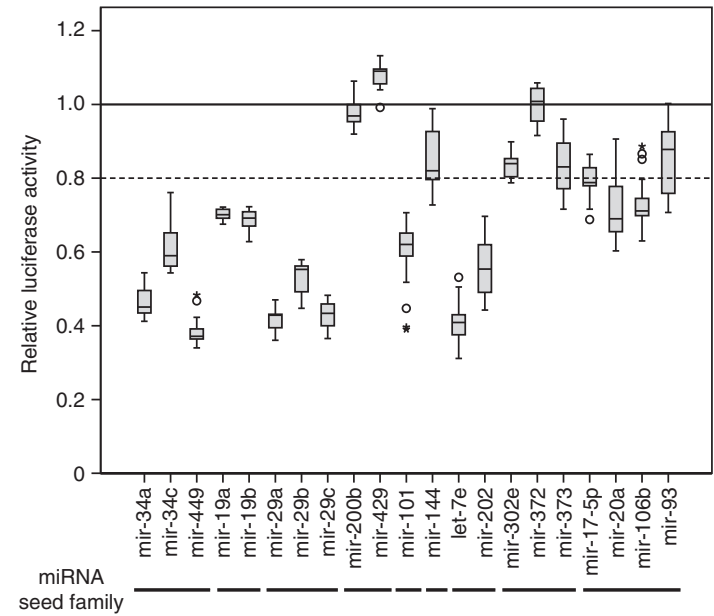

Figure 2 Prediction and experimental investigation of MYCN-targeting miRNAs. (A) Intersection of three web-based miRNA target prediction programmes (TargetScan, PicTar and miRanda) predicted 13 conserved binding sites for miRNAs broadly conserved among vertebrates. A total of 21 individual miRNAs were selected for target site validation. (B) Schematic overview of MYCN 3'UTR showing the localisation of the predicted miRNA target sites investigated in luciferase reporter assays (LRAs). (C) Results from LRAs shown as boxplot diagrams. Horizontal bars indicate miRNAs predicted to target identical target sites (miRNA seed families). Firefly luciferase was normalised to Renilla luciferase activity, and then normalised to the median activity of the control miRNA. Each box represents the distribution of the activity measured for a single miRNA (at least 9 individual transfections, range 9-36). Ends of boxes define the 25th and 75th percentile, a line defines the median, and bars define the lowest and highest value except outliers and extreme values, which are indicated by circles and asterisks, respectively. Reduction in relative luciferase activity of $\geqslant 20 \%$ was defined to be indicative for a miRNA/MYCN-3'UTR interaction.

miRNA binding sites in the pMIR-MYCN-UTR vector to abolish miRNA binding (Supplementary Figure 1). For miRNAs with two potential binding sites, both seeds were mutated individually (' $\mathrm{m} 1$ ' and ' $\mathrm{m} 2$ ', where ' $\mathrm{m} 1$ ' is the $5^{\prime}$-most binding site) as well as combined (' $\mathrm{m} 1+2$ '). We then performed LRAs with the 14 luciferase-repressing miRNAs, and compared the luciferase activities from the respective UTR-mutated and wild-type reporters. As shown in Figure 3, a rescue in luciferase activity was observed for several mutated target sites, indicating specific miRNA/MYCN-3'UTR interactions. Specifically, mir-34a/-34c/-449
(Figure 3A), mir-19a/-19b (Figure 3B), mir-29a/-29b/-29c (Figure 3C), mir-101 (Figure 3D) and let-7e/mir-202 (Figure 3F) were found to target the $M Y C N-3^{\prime}$ UTR sequence at positions $581-587$ (seed 2), 32-38, 334-340, 494-500 and 563-569 (seeds 1 and 2) and 870-876 (seed 2), respectively. We were not able to confirm specific targeting of mir-17/-20a/-106b to the predicted target site at position $859-865$ (Figure $3 \mathrm{E}$ ). An alternative binding site for mir-17/-20a/-106b at position 685-690 was uniquely predicted by the MiRanda software. However, mutation of this target site did not substantially affect the luciferase activity (Supplementary Figure 2a).

To exclude the possibility that the lack of luciferase rescue was because of incomplete destruction of target sites, we extended the mismatch from 2 to $7 \mathrm{nt}$ (complete seed mismatch) on selected target sites (mir-17/-20a/-106b and mir-34a/-34c/-449 - Supplementary Figure 1). However, the luciferase activities remained unaffected (Supplementary Figures $2 \mathrm{~b}$ and $\mathrm{c}$ ).

In summary, our data reveal six conserved miRNA binding sites in the $3^{\prime} \mathrm{UTR}$ structure of $M Y C N$ (Figure 3G). We were able to experimentally validate mir-34a, $-34 c,-449,-19 a,-19 b,-29 a,-29 b$, $-29 c,-101,-202$ and let-7e as MYCN-targeting miRNAs.

\section{The rs922 SNP does not affect miRNA binding}

The mir-150 is the only miRNA predicted to bind in close proximity to the rs922 SNP (Figure 1A, Supplementary Table 2). Overexpression of mir-150 in the LRA did not reduce luciferase expression from pMIR-MYCN-UTR or a mutated luciferase reporter containing the rs922 SNP (Supplementary Figure 3a). We then investigated whether the confirmed MYCN-targeting miRNAs were influenced by the rs922 SNP. We did not observe substantial luciferase rescue for the validated $M Y C N$-targeting miRNAs when the rs922-containing luciferase reporter was compared with the $3^{\prime}$ UTR wild-type sequence (Supplementary Figure 3a). This indicates that the rs922 SNP does not significantly influence miRNA-mediated suppression of MYCN expression.

\section{MiRNA-mediated MYCN suppression in MNA Kelly cells}

As the tumour-suppressor function of mir-34a and $-34 c$ in MNA neuroblastoma is well documented (Welch et al, 2007; Cole et al, 2008; Wei et al, 2008; Tivnan et al, 2011), we selected the remaining eight experimentally validated $M Y C N$-targeting miRNAs (mir-19a, -19b, -29a, -29b, -29c, -101, -202 and let-7e) for further functional analyses.

To investigate the capability of the selected miRNAs to downregulate endogenous N-myc protein in MNA neuroblastoma, we performed transient miRNA overexpression experiments and analysed N-myc expression by western blotting. As shown in Figure 4A, all eight miRNAs reduced endogenous $\mathrm{N}$-myc protein expression in Kelly cells. Particularly, let-7e and mir-202 were strong negative regulators of MYCN expression.

\section{Let-7e and mir-101 inhibit cell proliferation and clonogenic growth of MNA Kelly cells}

To investigate the functional role of $M Y C N$-targeting miRNAs in MNA neuroblastoma, we continuously monitored the proliferation of Kelly cells after transfection with miRNA mimics. As shown in Figure $4 \mathrm{~B}$, proliferation was significantly impaired when cells were transfected with mir-101 and let-7e. Mir-202, which targets the same seed sequence as let-7e, suppressed proliferation of Kelly cells similar to that observed for let-7e (Supplementary Figure 4). Cell proliferation was not substantially altered when mir-19a/b or mir-29a/b/c were overexpressed in Kelly cells (data not shown).

We further investigated the long-term effect of transient let-7e and mir-101 overexpression on clonogenic cell growth. Kelly cells were transfected with let-7e and mir-101 miRNA mimics and 

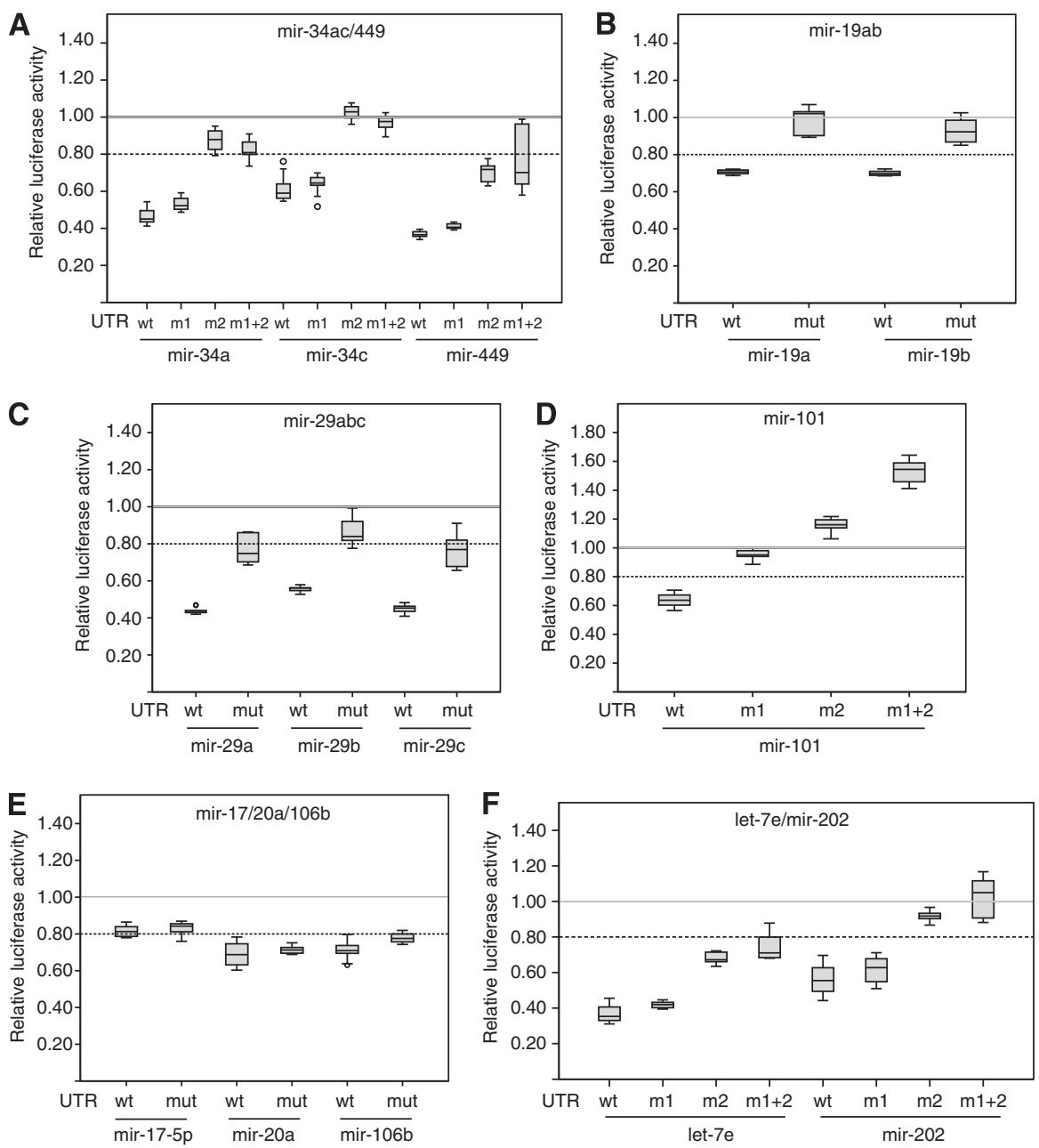

G

Experimentally validated miRNA target sites in the MYCN 3'UTR (909bp)

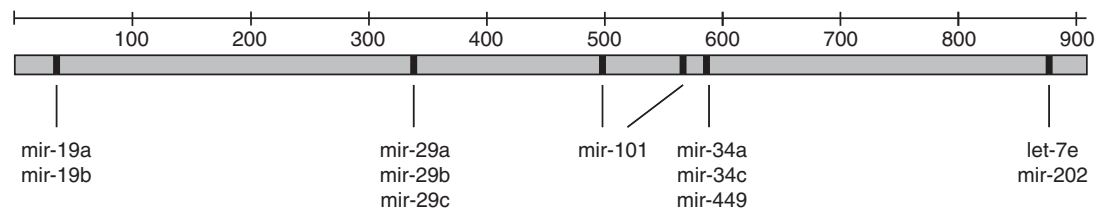

Figure 3 Experimental validation of miRNA target sites in the $3^{\prime} U T R$ sequence of MYCN. The predicted target sites for the miRNA families mir-34al-cl449 (A), mir-19al-b (B), mir-29al-b/-c (C), mir-101 (D), mir-17/-20al-106b (E) and let-7/mir-202 (F) were mutated using site-directed mutagenesis. $w t=$ wild-type $3^{\prime} U T R$ sequence; mut $=3^{\prime} \cup T R$ sequences mutated in the seed sequence corresponding to the investigated miRNA (Supplementary Figure I). For miRNA families containing two potential target sites, ' $\mathrm{ml}$ ' and ' $\mathrm{m} 2$ ' denote mutations of the 5'-most and 3'-most target site, respectively. ' $\mathrm{ml}+2$ ' means that both target sites are mutated. The target site mutants were investigated in LRAs and compared with the wild-type MYCN-3'UTR sequence. Graphical presentations are similar to that described for Figure 2. (G) Overview of the six experimentally confirmed miRNA target sites in the MYCN $3^{\prime} U T R$. The individual miRNAs used in the validation experiments are shown.

plated at 200 cells per well in six-well tissue culture plates. Visible cell clones were counted after 2 weeks of incubation. Compared with the negative control mimic, overexpression of let-7e and mir-101 in Kelly cells reduced colony formation by $80 \%$ and $50 \%$, respectively (Figure $4 \mathrm{C}$ ).

In conclusion, we established that the let-7 family miRNAs, let-7e and mir-202, and mir-101 have strong antiproliferative properties in the MNA neuroblastoma cell line Kelly.

\section{DISCUSSION}

Deregulated MYCN expression is a hallmark in high-risk neuroblastoma. Here, we aimed to investigate how miRNAs contribute to $M Y C N$ regulation. We systematically investigated the $M Y C N-3^{\prime}$ UTR sequence for potential miRNA binding sites. We used LRAs to show that the $3^{\prime}$ UTR sequence is directly targeted by several miRNAs (mir-34a, -34c, $-449,-19 a,-19 b,-29 a,-29 b,-29 c$, $-101,-202$ and let-7e). These miRNAs were further shown to decrease $\mathrm{N}$-myc protein expression when overexpressed in the MNA neuroblastoma cell line Kelly. Finally, we showed that let-7e, mir-101 and mir-202 efficiently inhibit proliferation and clonogenic cell growth in Kelly cells.

It has been reported that certain mutations and SNPs in the $3^{\prime}$ UTR of cancer-related genes increase cancer susceptibility and may allow the cancer cell to escape miRNA regulation (Pelletier and Weidhaas, 2010). Here, we show that mutations in the MYCN $3^{\prime}$ UTR are rare, both in MNA and non-MNA neuroblastoma cells. 
A
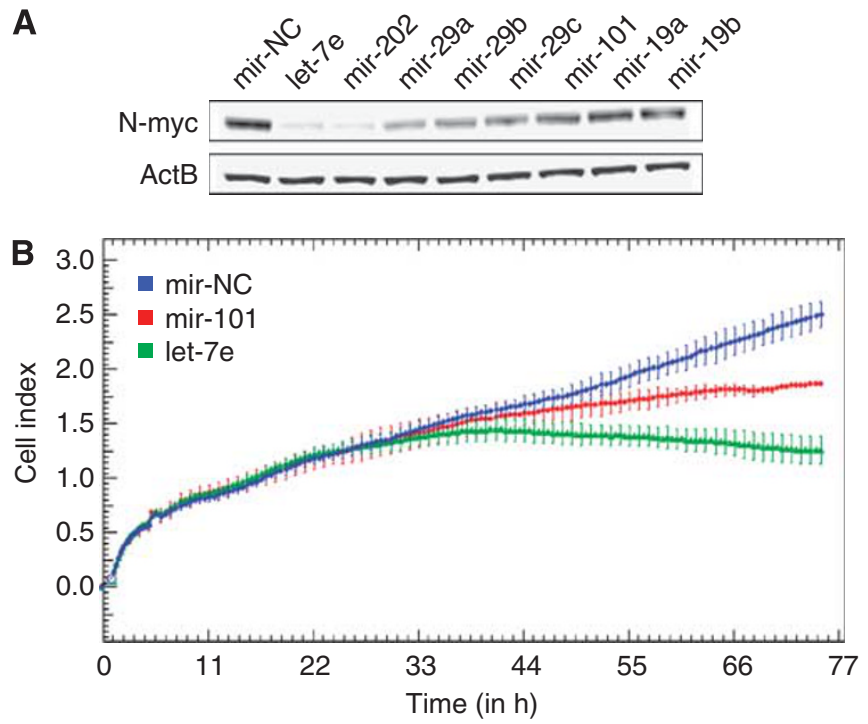

C

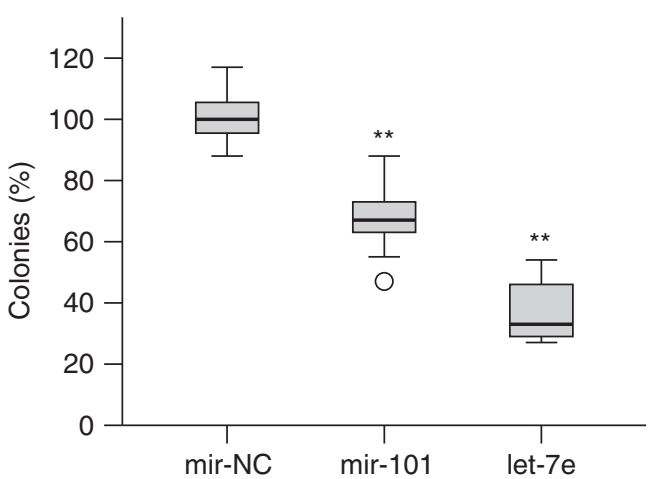

Figure 4 The miRNA-mediated N-Myc downregulation and growth inhibition in MNA Kelly cells. (A) A representative western blot showing strong N-Myc protein reduction upon transfection of let-7e, mir-202, mir-29al-b/-c and mir-101. Mir-19a/-b reduced N-Myc levels only slightly. (B) The 72-h continuous monitoring of cell proliferation after transfection of let-7e, mir-10I or a negative control (mir-NC) mimic into Kelly cells. (C) Clonogenic growth of Kelly cells was significantly impaired after transfection of let-7e and mir-101 mimics, compared with the negative control (** $P<0.001$, Student's $t$-test). Each boxplot represents the distribution of colony numbers from three independent transfections, normalised to the median number of the negative control.

We detected a single SNP (rs922) at position 250 (C250T) of the $3^{\prime}$ UTR, which did not impair miRNA binding. We were unable to detect heterozygous $\mathrm{C} / \mathrm{T}$ variants in our samples, which might be explained by the fact that only one allele is amplified in MNA samples, and by the limited number of investigated non-MNA samples.

The mir-19a and mir-19b, which are both expressed from the oncogenic mir-17-92 cluster, were validated to target the MYCN $3^{\prime}$ UTR in the luciferase reporter assay system in HEK293 cells. However, we were not able to demonstrate a significant N-Myc protein downregulation after overexpression in Kelly cells. As $M Y C N$ itself is a direct activator of the mir-17-92 cluster (Loven et $a l, 2010)$, we assume that the $M Y C N$-targeting function of mir-19a/-b is disturbed in MNA neuroblastoma cells.

The mir-34 family consists of mir-34a, $-34 b$ and $-34 c$. The mir-34a is encoded by a locus at chromosome 1p36, whereas mir-34b and $-34 c$ are coexpressed from a locus at chromosome 11q23. Both regions are commonly hemizygously deleted in neuroblastoma (Attiyeh et al, 2005). In contrast to mir-34b, both mir-34a and mir-34c have been shown to inhibit cell growth in several neuroblastoma cell lines with 1 p36 hemizygous deletion (Welch et al, 2007; Cole et al, 2008; Wei et al, 2008). The mir-34a has several experimentally validated targets involved in cellular proliferation, including MYCN (Hermeking, 2010). In this study, we confirm that mir-34a directly targets the $3^{\prime} \mathrm{UTR}$ sequence of MYCN. Whereas Wei et al (2008) concluded that both mir-34 target sites were required to obtain maximum $M Y C N$ suppression by mir-34a, we found the $3^{\prime}$-most target site (position 581-587) alone to be responsible for most of the suppressive effect. Not even complete destruction of the $5^{\prime}$-most target site (position 23-29) could significantly rescue luciferase activity to indicate that this site is involved in mir-34a-mediated repression of MYCN (Supplementary Figure 2). This result is supported by data from a study performed by Stallings and co-workers (Welch et al, 2007), who were unable to show mir-34a-mediated suppression of a luciferase reporter containing only the $5^{\prime}$-most mir-34 target site. The observed discrepancy could be explained by the different $3^{\prime}$ UTR sequences and cells used in the LRAs. Whereas we used the full-length 909 nt $M Y C N-3^{\prime}$ UTR sequence and HEK293 cells, Wei et al (2008) used a longer sequence including additional $418 \mathrm{nt}$ of the MYCN coding sequence and SK-N-AS cells. We also verified that mir-34c and mir-449, but not mir-34b, target the MYCN $3^{\prime} \mathrm{UTR}$ similar to mir-34a (Figures $2 \mathrm{C}$ and $3 \mathrm{~A}$, Supplementary Figure 2 and data not shown).

In a study to validate the TargetScan algorithm, mir-101 was predicted to potentially target two sequences in the MYCN $3^{\prime}$ UTR (Lewis et al, 2003). The 5'-most target site (position 495-500) was mutated in a $409 \mathrm{nt} 3^{\prime} \mathrm{UTR}$ fragment coupled to a luciferase reporter to verify it as a target of endogenous mir-101 in HeLa cells. Here, we confirm mir-101 as a MYCN-regulating miRNA able to suppress $M Y C N$ expression from both predicted target sites (Figures 2C and 3D). In addition, we show that mir-101 inhibits proliferation of MNA Kelly cells. These data from MNA neuroblastoma confirm and extend previous reports showing tumour-suppressor properties of mir-101 in different other cancer types (Varambally et al, 2008; Strillacci et al, 2009; Su et al, 2009; Cao et al, 2010; Wang et al, 2010; Zhang et al, 2011).

$\mathrm{Xu}$ et al (2009) have previously shown that mir-29 directly regulates $\mathrm{B} 7-\mathrm{H} 3$, a surface glycoprotein of the B7/CD28 family that is expressed on a wide variety of solid tumour cells, including neuroblastoma. B7-H3 has immunoinhibitory effects protecting neuroblastoma cells from NK-mediated cytotoxicity (Castriconi et al, 2004). In addition, B7-H3 is the target of the monoclonal antibody $8 \mathrm{H} 9$ (Xu et al, 2009) that showed promising results when used in compartmental radioimmunotherapy (cRIT) in a clinical trial for CNS-relapsed high-risk neuroblastoma (Kramer et al, 2010). Compared with normal tissue, mir-29 was found significantly lower expressed in neuroblastoma cells, contributing to a higher expression of $\mathrm{B} 7-\mathrm{H} 3$ on neuroblastoma cell surfaces (Xu et al, 2009). It has been suggested that restoration of mir-29 and subsequent translational inhibition of B7-H3 might therefore prove therapeutically beneficial, both by sensitising neuroblastoma cells to NK/T-cell-mediated immunotoxicity and by protecting B7-H3 expressing normal tissue from 8H9-related toxicity (Xu et al, 2009). Our data extend the therapeutical potential of mir-29 as it was shown to directly target MYCN.

The human let-7 miRNA family consists of 10 different mature let-7 sequences that are derived from 13 precursors (Roush and Slack, 2008). Overexpression of let-7 has been shown to inhibit proliferation of breast cancer (Zhao et al, 2011), lung cancer (Johnson et al, 2007), prostate cancer (Dong et al, 2010), colon cancer (Akao et al, 2006), malignant melanoma (Schultz et al, 2008) and glioblastoma (Lee et al, 2011) cell lines. Several important cell cycle regulators, including cyclins, cyclin-dependent kinases (CDKs), Ras, HMGA2 and c-Myc have previously been confirmed to be targets of let-7 (Johnson et al, 2005, 2007; Lee and Dutta, 2007; Schultz et al, 2008; Kim et al, 2009). We have now 
added the MYCN oncogene to the list of cell cycle regulators targeted by let-7. The observed growth-inhibitory effect of let-7e on MNA neuroblastoma cells is most likely because of the combined suppression of several let-7 targets involved in cell proliferation.

In summary, we were able to define a subset of miRNA that are able to regulate $M Y C N$ expression when overexpressed in MNA neuroblastoma cells. To what extent the $\mathrm{N}$-myc protein is regulated by endogenous levels of these miRNAs, and if altered levels contribute to neuroblastoma development, needs to be addressed in further studies. Recent data from miRNA profiling studies show that let-7e, mir-29a and mir-29c are significantly lower expressed in MNA primary tumours compared with non-MNA tumours

\section{REFERENCES}

Akao Y, Nakagawa Y, Naoe T (2006) let-7 microRNA functions as a potential growth suppressor in human colon cancer cells. Biol Pharm Bull 29(5): $903-906$

Albihn A, Johnsen JI, Henriksson MA (2010) MYC in oncogenesis and as a target for cancer therapies. Adv Cancer Res 107: 163-224

Attiyeh EF, London WB, Mosse YP, Wang Q, Winter C, Khazi D, McGrady PW, Seeger RC, Look AT, Shimada H, Brodeur GM, Cohn SL, Matthay KK, Maris JM (2005) Chromosome 1p and 11q deletions and outcome in neuroblastoma. N Engl J Med 353(21): 2243-2253

Bell E, Chen L, Liu T, Marshall GM, Lunec J, Tweddle DA (2010) MYCN oncoprotein targets and their therapeutic potential. Cancer Lett 293(2): $144-157$

Buechner J, Henriksen JR, Haug BH, Tomte E, Flaegstad T, Einvik C (2011) Inhibition of mir-21, which is up-regulated during MYCN knockdownmediated differentiation, does not prevent differentiation of neuroblastoma cells. Differentiation 81(1): $25-34$

Cao P, Deng Z, Wan M, Huang W, Cramer SD, Xu J, Lei M, Sui G (2010) MicroRNA-101 negatively regulates Ezh2 and its expression is modulated by androgen receptor and HIF-1alpha/HIF-1beta. Mol Cancer 9: 108

Castriconi R, Dondero A, Augugliaro R, Cantoni C, Carnemolla B, Sementa AR, Negri F, Conte R, Corrias MV, Moretta L, Moretta A, Bottino C (2004) Identification of $4 \mathrm{Ig}-\mathrm{B} 7-\mathrm{H} 3$ as a neuroblastoma-associated molecule that exerts a protective role from an NK cell-mediated lysis. Proc Natl Acad Sci USA 101(34): 12640-12645

Cohn SL, London WB, Huang D, Katzenstein HM, Salwen HR, Reinhart T, Madafiglio J, Marshall GM, Norris MD, Haber M (2000) MYCN expression is not prognostic of adverse outcome in advanced-stage neuroblastoma with nonamplified MYCN. J Clin Oncol 18(21): 3604-3613

Cole KA, Attiyeh EF, Mosse YP, Laquaglia MJ, Diskin SJ, Brodeur GM, Maris JM (2008) A functional screen identifies miR-34a as a candidate neuroblastoma tumor suppressor gene. Mol Cancer Res 6(5): 735-742

Doench JG, Sharp PA (2004) Specificity of microRNA target selection in translational repression. Genes Dev 18(5): 504-511

Dong Q, Meng P, Wang T, Qin W, Wang F, Yuan J, Chen Z, Yang A, Wang $H$ (2010) MicroRNA let-7a inhibits proliferation of human prostate cancer cells in vitro and in vivo by targeting E2F2 and CCND2. PLoS One 5(4): e10147

Edsjo A, Nilsson H, Vandesompele J, Karlsson J, Pattyn F, Culp LA, Speleman F, Pahlman S (2004) Neuroblastoma cells with overexpressed MYCN retain their capacity to undergo neuronal differentiation. Lab Invest 84(4): $406-417$

Henriksen JR, Haug BH, Buechner J, Tomte E, Lokke C, Flaegstad T, Einvik C (2011) Conditional expression of retrovirally delivered antiMYCN shRNA as an in vitro model system to study neuronal differentiation in MYCN-amplified neuroblastoma. BMC Dev Biol 11: 1

Hermeking H (2010) The miR-34 family in cancer and apoptosis. Cell Death Diff 17(2): $193-199$

Hooker CW, Hurlin PJ (2006) Of Myc and Mnt. J Cell Sci 119(Pt 2): $208-216$

Iraci N, Diolaiti D, Papa A, Porro A, Valli E, Gherardi S, Herold S, Eilers M, Bernardoni R, Della Valle G, Perini G (2011) A SP1/MIZ1/MYCN repression complex recruits HDAC1 at the TRKA and p75NTR promoters and affects neuroblastoma malignancy by inhibiting the cell response to NGF. Cancer Res 71(2): 404-412

John B, Enright AJ, Aravin A, Tuschl T, Sander C, Marks DS (2004) Human microRNA targets. PLoS Biol 2: e363
(Schulte et al, 2010) (Supplementary Figure 5), supporting the idea that they act as endogenous MYCN regulators.

\section{ACKNOWLEDGEMENTS}

This work was supported by grants from the Northern-Norwegian Health Authorities (gene therapy program) and the Norwegian Cancer Society (Ragnvarda F. Sørvik and Håkon Starheims Foundation).

Supplementary Information accompanies the paper on British Journal of Cancer website (http://www.nature.com/bjc)

Johnson CD, Esquela-Kerscher A, Stefani G, Byrom M, Kelnar K, Ovcharenko D, Wilson M, Wang X, Shelton J, Shingara J, Chin L, Brown D, Slack FJ (2007) The let-7 microRNA represses cell proliferation pathways in human cells. Cell Res 67(16): 7713-7722

Johnson SM, Grosshans H, Shingara J, Byrom M, Jarvis R, Cheng A, Labourier E, Reinert KL, Brown D, Slack FJ (2005) RAS is regulated by the let-7 microRNA family. Cell 120(5): 635-647

Kim HH, Kuwano Y, Srikantan S, Lee EK, Martindale JL, Gorospe M (2009) $\mathrm{HuR}$ recruits let-7/RISC to repress c-Myc expression. Genes Dev 23(15): $1743-1748$

Kohl NE, Legouy E, DePinho RA, Nisen PD, Smith RK, Gee CE, Alt FW (1986) Human N-myc is closely related in organization and nucleotide sequence to c-myc. Nature 319(6048): $73-77$

Kramer K, Kushner BH, Modak S, Pandit-Taskar N, Smith-Jones P, Zanzonico P, Humm JL, Xu H, Wolden SL, Souweidane MM, Larson SM, Cheung NK (2010) Compartmental intrathecal radioimmunotherapy: results for treatment for metastatic CNS neuroblastoma. J Neuroonco 97(3): $409-418$

Lall S, Grun D, Krek A, Chen K, Wang YL, Dewey CN, Sood P, Colombo T, Bray N, Macmenamin P, Kao HL, Gunsalus KC, Pachter L, Piano F, Rajewsky N (2006) A genome-wide map of conserved microRNA targets in $C$ elegans. Curr Biol 16: 460-471

Larsson LG, Henriksson MA (2010) The Yin and Yang functions of the Myc oncoprotein in cancer development and as targets for therapy. Exp Cell Res 316(8): $1429-1437$

Lee ST, Chu K, Oh HJ, Im WS, Lim JY, Kim SK, Park CK, Jung KH, Lee SK, Kim M, Roh JK (2011) Let-7 microRNA inhibits the proliferation of human glioblastoma cells. J Neurooncol 102(1): 19-24

Lee YS, Dutta A (2007) The tumor suppressor microRNA let-7 represses the HMGA2 oncogene. Genes Dev 21(9): 1025-1030

Lewis BP, Burge CB, Bartel DP (2005) Conserved seed pairing, often flanked by adenosines, indicates that thousands of human genes are microRNA targets. Cell 120(1): 15-20

Lewis BP, Shih IH, Jones-Rhoades MW, Bartel DP, Burge CB (2003) Prediction of mammalian microRNA targets. Cell 115(7): $787-798$

Liu T, Tee AE, Porro A, Smith SA, Dwarte T, Liu PY, Iraci N, Sekyere E, Haber M, Norris MD, Diolaiti D, Della Valle G, Perini G, Marshall GM (2007) Activation of tissue transglutaminase transcription by histone deacetylase inhibition as a therapeutic approach for Myc oncogenesis. Proc Natl Acad Sci USA 104(47): $18682-18687$

Loven J, Zinin N, Wahlstrom T, Muller I, Brodin P, Fredlund E, Ribacke U, Pivarcsi A, Pahlman S, Henriksson M (2010) MYCN-regulated microRNAs repress estrogen receptor-alpha (ESR1) expression and neuronal differentiation in human neuroblastoma. Proc Natl Acad Sci USA 107(4): $1553-1558$

Maris JM (2010) Recent advances in neuroblastoma. N Engl J Med 362(23): $2202-2211$

Maris JM, Hogarty MD, Bagatell R, Cohn SL (2007) Neuroblastoma. Lancet 369(9579): $2106-2120$

Meyer N, Penn LZ (2008) Reflecting on 25 years with MYC. Nat Rev Cancer 8(12): $976-990$

Nilsson JA, Cleveland JL (2003) Myc pathways provoking cell suicide and cancer. Oncogene 22(56): $9007-9021$

Pelletier C, Weidhaas JB (2010) MicroRNA binding site polymorphisms as biomarkers of cancer risk. Expert Rev Mol Diagn 10(6): 817-829 
Roush S, Slack FJ (2008) The let-7 family of microRNAs. Trends Cell Biol 18(10): $505-516$

Schulte JH, Schowe B, Mestdagh P, Kaderali L, Kalaghatgi P, Schlierf S, Vermeulen J, Brockmeyer B, Pajtler K, Thor T, de Preter K, Speleman F, Morik K, Eggert A, Vandesompele J, Schramm A (2010) Accurate prediction of neuroblastoma outcome based on miRNA expression profiles. Int J Cancer 127(10): 2374-2385

Schultz J, Lorenz P, Gross G, Ibrahim S, Kunz M (2008) MicroRNA let-7b targets important cell cycle molecules in malignant melanoma cells and interferes with anchorage-independent growth. Cell Res 18(5): 549-557

Siomi H, Siomi MC (2009) On the road to reading the RNA-interference code. Nature 457(7228): 396-404

Stallings RL, Foley NH, Bryan K, Buckley PG, Bray I (2010) Therapeutic targeting of miRNAs in neuroblastoma. Expert Opin Ther Targets 14(9): $951-962$

Stanton BR, Perkins AS, Tessarollo L, Sassoon DA, Parada LF (1992) Loss of $\mathrm{N}$-myc function results in embryonic lethality and failure of the epithelial component of the embryo to develop. Genes Dev 6(12A): 2235-2247

Strillacci A, Griffoni C, Sansone P, Paterini P, Piazzi G, Lazzarini G, Spisni E, Pantaleo MA, Biasco G, Tomasi V (2009) MiR-101 downregulation is involved in cyclooxygenase- 2 overexpression in human colon cancer cells. Exp Cell Res 315(8): 1439-1447

Su H, Yang JR, Xu T, Huang J, Xu L, Yuan Y, Zhuang SM (2009) MicroRNA-101, down-regulated in hepatocellular carcinoma, promotes apoptosis and suppresses tumorigenicity. Cancer Res 69(3): 1135-1142

Tang XX, Zhao H, Kung B, Kim DY, Hicks SL, Cohn SL, Cheung NK, Seeger RC, Evans AE, Ikegaki N (2006) The MYCN enigma: significance of MYCN expression in neuroblastoma. Cancer Res 66(5): 2826-2833

Tivnan A, Tracey L, Buckley PG, Alcock LC, Davidoff AM, Stallings RL (2011) MicroRNA-34a is a potent tumor suppressor molecule in vivo in neuroblastoma. BMC Cancer 11: 33
Varambally S, Cao Q, Mani RS, Shankar S, Wang X, Ateeq B, Laxman B, Cao X, Jing X, Ramnarayanan K, Brenner JC, Yu J, Kim JH, Han B, Tan P, Kumar-Sinha C, Lonigro RJ, Palanisamy N, Maher CA, Chinnaiyan AM (2008) Genomic loss of microRNA-101 leads to overexpression of histone methyltransferase EZH2 in cancer. Science 322(5908): 1695-1699

Vita M, Henriksson M (2006) The Myc oncoprotein as a therapeutic target for human cancer. Semin Cancer Biol 16(4): 318-330

Wang HJ, Ruan HJ, He XJ, Ma YY, Jiang XT, Xia YJ, Ye ZY, Tao HQ (2010) MicroRNA-101 is down-regulated in gastric cancer and involved in cell migration and invasion. Eur J Cancer 46(12): 2295-2303

Wei JS, Song YK, Durinck S, Chen QR, Cheuk AT, Tsang P, Zhang Q, Thiele CJ, Slack A, Shohet J, Khan J (2008) The MYCN oncogene is a direct target of miR-34a. Oncogene 27(39): 5204-5213

Welch C, Chen Y, Stallings RL (2007) MicroRNA-34a functions as a potential tumor suppressor by inducing apoptosis in neuroblastoma cells. Oncogene 26(34): 5017-5022

Westermann F, Muth D, Benner A, Bauer T, Henrich KO, Oberthuer A, Brors B, Beissbarth T, Vandesompele J, Pattyn F, Hero B, Konig R, Fischer M, Schwab M (2008) Distinct transcriptional MYCN/c-MYC activities are associated with spontaneous regression or malignant progression in neuroblastomas. Genome Biol 9(10): R150

$\mathrm{Xu} \mathrm{H}$, Cheung IY, Guo HF, Cheung NK (2009) MicroRNA miR-29 modulates expression of immunoinhibitory molecule B7-H3: potential implications for immune based therapy of human solid tumors. Cancer Res 69(15): $6275-6281$

Zhang JG, Guo JF, Liu DL, Liu Q, Wang JJ (2011) MicroRNA-101 exerts tumorsuppressive functions in non-small cell lung cancer through directly targeting enhancer of Zeste homolog 2.J Thorac Oncol 6(4): 671-678

Zhao Y, Deng C, Wang J, Xiao J, Gatalica Z, Recker RR, Xiao GG (2011) Let-7 family miRNAs regulate estrogen receptor alpha signaling in estrogen receptor positive breast cancer. Breast Cancer Res Treat 127(1): 69-80 